\title{
Correction to: STIM1 deficiency is linked to Alzheimer's disease and triggers cell death in SH-SY5Y cells by upregulation of L-type voltage-operated $\mathrm{Ca}^{2+}$ entry
}

\author{
Carlos Pascual-Caro $^{1}$ - Maria Berrocal ${ }^{1}$ - Aida M. Lopez-Guerrero ${ }^{1}$ - Alberto Alvarez-Barrientos ${ }^{2}$. \\ Eulalia Pozo-Guisado ${ }^{3}$ - Carlos Gutierrez-Merino ${ }^{1}$ - Ana M. Mata ${ }^{1}$ • Francisco Javier Martin-Romero ${ }^{1}$
}

Published online: 2 July 2019

(C) Springer-Verlag GmbH Germany, part of Springer Nature 2019

\section{Correction to: Journal of Molecular Medicine (2018) 96:1061-1079} https://doi.org/10.1007/s00109-018-1677-y

The original publication of this paper contains errors.

Page 1066, first column, penultimate lane: where the text says " $\mathrm{Ca}^{2+}=0.76 \mathrm{mM}$ ", it should say " $\mathrm{Ca}^{2+}=0.76 \mu \mathrm{M}$ "

Page 1070, second column, two last lanes: where the text says "27.8 $\pm 22.8 \mu \mathrm{M}$ for $\mathrm{KO}$ vs $66.4 \pm 10.9 \mu \mathrm{M}$ for WT", it should say "27.8 $\pm 22.8 \mathrm{nM}$ for KO vs $66.4 \pm 10.9 \mathrm{nM}$ for WT"

Page 1073, y-axis in the bar chart of the panel b: where the text says "Mitochondrial $\left[\mathrm{Ca}^{2+}\right](\mu \mathrm{M})$ " it should say "Mitochondrial $\left[\mathrm{Ca}^{2+}\right](\mathrm{nM})$ "

The online version of the original article can be found at https://doi.org/ 10.1007/s00109-018-1677-y

Francisco Javier Martin-Romero

fjmartin@unex.es

Carlos Pascual-Caro

carlospc@unex.es

Maria Berrocal

mabeca@unex.es

Aida M. Lopez-Guerrero

ailogue@unex.es

Alberto Alvarez-Barrientos

alalvarezb@unex.es

Eulalia Pozo-Guisado

epozo@unex.es
Page 1074, y-axis in the bar chart of the panel e: where the text says "Mitochondrial $\left[\mathrm{Ca}^{2+}\right](\mu \mathrm{M})$ " it should say "Mitochondrial $\left[\mathrm{Ca}^{2+}\right](\mathrm{nM})$ "

Page 1074, second column, fifth lane: where the text says "46 $\mu \mathrm{M}$ ", it should say "46 nM"

Because the y-axis label in two different bar chart should be corrected, we provide the full figures with the corrected labels.

Publisher's note Springer Nature remains neutral with regard to jurisdictional claims in published maps and institutional affiliations.

Carlos Gutierrez-Merino

carlosgm@unex.es

Ana M. Mata

anam@unex.es

1 Department of Biochemistry and Molecular Biology, School of Life Sciences and Institute of Molecular Pathology Biomarkers, University of Extremadura, Avenida de Elvas s/n, 06006 Badajoz, Spain

2 Applied Bioscience Facility, University of Extremadura, Avenida de Elvas s/n, 06006 Badajoz, Spain

3 Department of Cell Biology, School of Medicine and Institute of Molecular Pathology Biomarkers, University of Extremadura, Avenida de Elvas s/n, 06006 Badajoz, Spain 
a

\begin{tabular}{|c|c|c|}
\hline & \multicolumn{2}{|c|}{$\begin{array}{l}\text { NADH oxidase activity } \\
\left(\mathrm{nmol} x \mathrm{mg} \text { proten }{ }^{-1} \times \mathrm{min}^{-1}\right)\end{array}$} \\
\hline & Total activity & Rotenone-insensitive \\
\hline Wild-type & $9.7 \pm 0.7$ & $6.82 \pm 1.3$ \\
\hline STIM1-KO & $6.85 \pm 0.75$ & $6.1 \pm 1.2$ \\
\hline
\end{tabular}

b

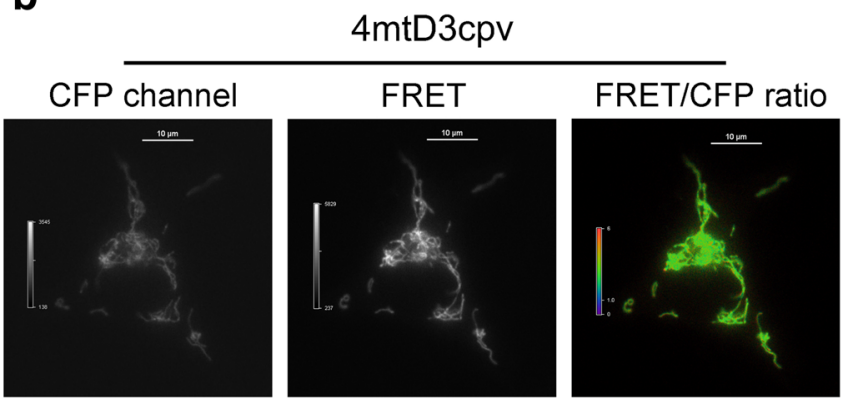

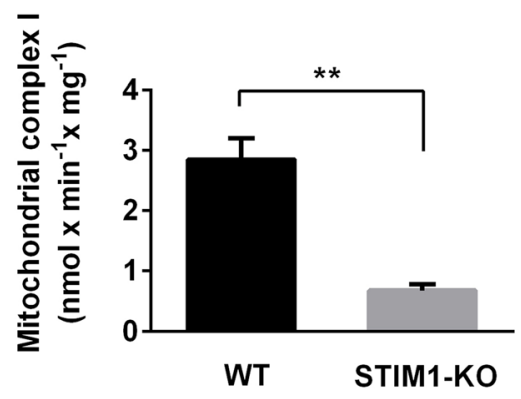

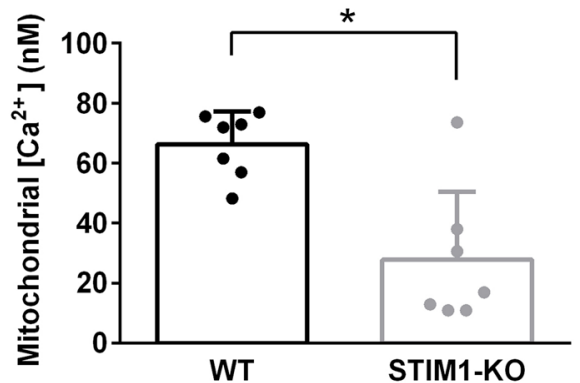

Fig. 7 Mitochondrial electron transport complex and mitochondrial $\mathrm{Ca}^{2+}$ levels. a Total NADH oxidase activity and rotenone-sensitive activity was assessed from differentiated SH-SY5Y cell lysates (WT and STIM1-KO). Data are presented as the mean \pm s.d. of two independent experiments. Right panel shows the difference between total activity and the remaining activity after rotenone addition to the assay, i.e., the rotenone-sensitive NADH oxidase. b Wild-type and STIM1-KO cells were transiently transfected for the expression of the $\mathrm{Ca}^{2+}$ sensor $4 \mathrm{mtD} 3 \mathrm{cpv}$ and $48 \mathrm{~h}$ later emission of fluorescence was recorded for CFP, FRET (left and middle panels,), and YFP channels to monitor photobleaching. FRET/CFP ratio signal (right panel) was recorded for cells in $\mathrm{Ca}^{2+}$-containing HBSS for 4-5 min. Calibration of FRET/CFP ratio to calculate Rmin and Rmax was performed individually for every assay. $\left[\mathrm{Ca}^{2+}\right]_{\mathrm{m}}$ data are presented as the mean \pm s.d. of seven independent experiments 
a

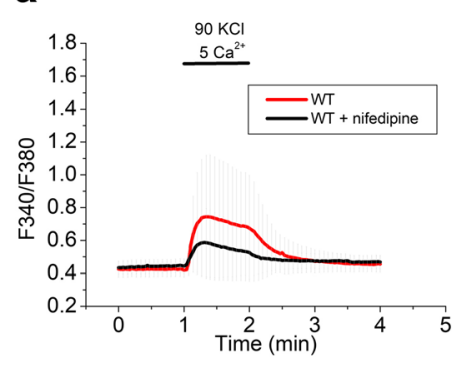

b

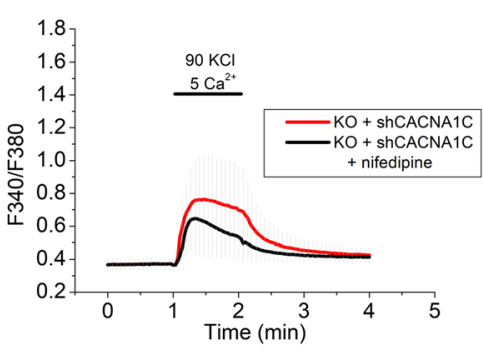

C

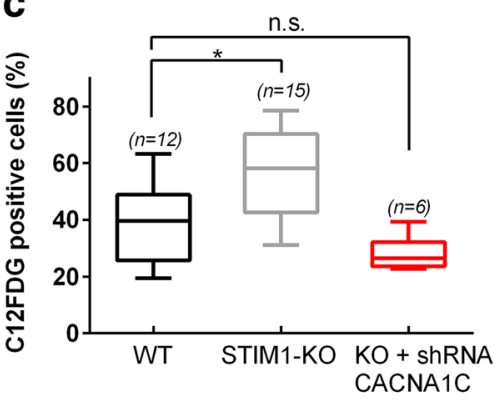

d

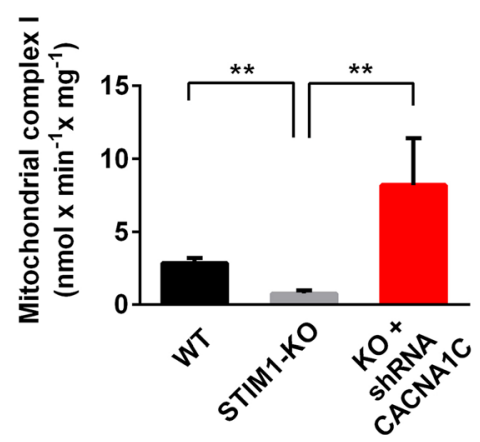

Control $\omega$-conotoxin MVIIC

nifedipine $\mathrm{ML} 218$
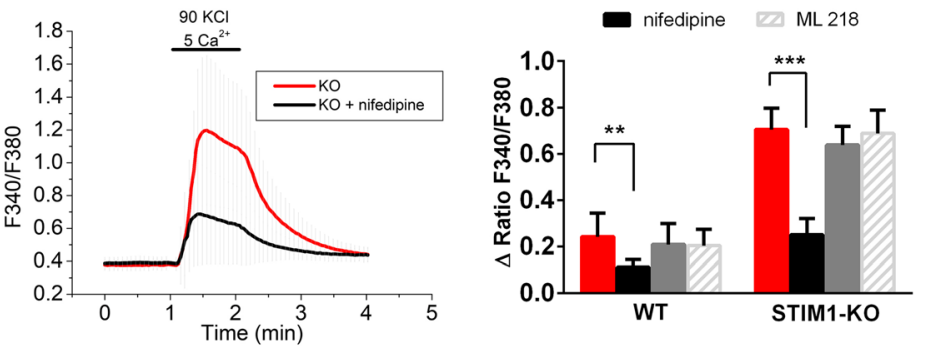
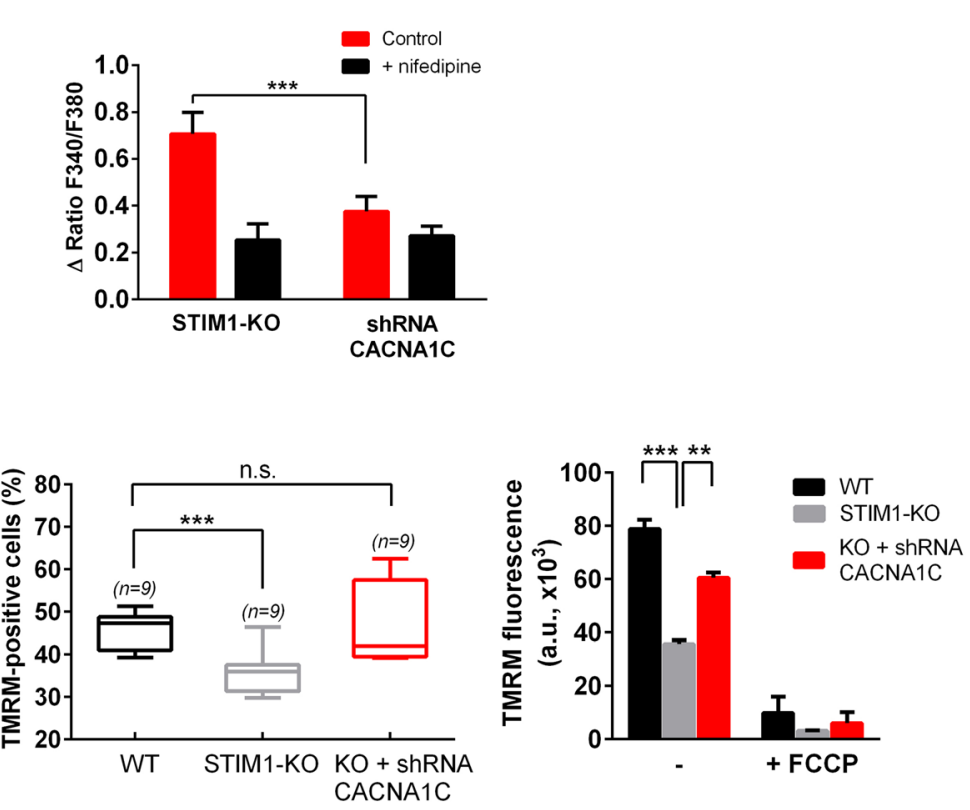

e

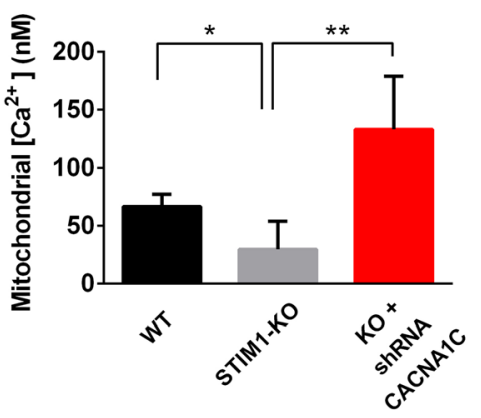

Fig. 8 Increased cellular $\mathrm{Ca}^{2+}$ influx underlies mitochondrial failure and augmented senescence. a Changes in cytosolic-free $\mathrm{Ca}^{2+}$ concentration were analyzed in fura-2-loaded cells. Cells in HBSS containing $1.26 \mathrm{mM}$ $\mathrm{Ca}^{2+}$ were subjected to 1-min depolarization with $90 \mathrm{mM} \mathrm{KCl}$ (red line). $\mathrm{CaCl}_{2}$ in the HBSS was increased to $5 \mathrm{mM}$ during depolarization to facilitate the $\mathrm{Ca}^{2+}$ influx recording. In parallel experiments, $10 \mu \mathrm{M}$ nifedipine was added to the assay medium during the recording (black line). Right panel: the increase of the $\mathrm{F} 340 / \mathrm{F} 380$ ratio triggered by $90 \mathrm{mM} \mathrm{KCl}$ in the presence of VOCCs blockers is shown as mean \pm s.d. of 3 experiments (a minimum of 70 cells per experimental condition). Final concentrations: $10 \mu \mathrm{M}$ nifedipine, $1 \mu \mathrm{M} \omega$-conotoxin MVIIC, $3 \mu \mathrm{M}$ ML 218. b STIM1-KO cells, or STIM1-KO cells stably expressing a specific shRNA to knock-down CACNAIC transcripts, were treated as described in panel (a). The left panel shows a representative experiment, and the bar

chart of the right panel shows the increase in the F340/F380 ratio evoked by depolarization (mean \pm s.d. of two independent experiments; $n>60$ cells per condition). c Senescence (left panel) and mitochondrial polarization (middle and right panels) were assessed from differentiated cells after 6 DIV, staining with C12FDG as described in Fig. 5c and TMRM as in Fig. $6 \mathrm{~b}-\mathrm{d}$, respectively. Data are mean \pm s.d. of three independent experiments (number of replicates is shown for each condition). d Rotenone-sensitive NADH oxidase activity was assessed from differentiated SH-SY5Y cell lysates (wild-type, STIM1-KO, and STIM1-KO + shRNA for CACNA1C). Data are presented as the mean \pm s.d. of two independent experiments. $\mathbf{e}$ Cell were transiently transfected for the expression of the $\mathrm{Ca}^{2+}$ sensor $4 \mathrm{mtD} 3 \mathrm{cpv}$. Mitochondrial $\left[\mathrm{Ca}^{2+}\right]$ was assessed as described in Fig. 7. Data of six independent experiments are shown in the right panel bar chart as mean \pm s.d. 